\title{
Un bel exemple d'entraide : le protéolipide PLP secouru par son isoforme, DM20, dans l'élaboration de la myéline
}

La protéine protéolipidique (PI.P), constituant majeur de la myéline dans le système nerveux, est une des protéines les mieux conservées au cours de l'évolution ( $100 \%$ d'identité entre la PLP humaine et la Plp murine). Les mutations animales, qui sont nombreuses, offrent donc d'excellents modèles d'étude $(\mathrm{m} / \mathrm{s}$ $n^{\circ} 1$, vol. 6, p. 70). La mutation Jimpy (au site accepteur de l'intron 4), dont il résulte une protéine tronquée ( $m / s n^{\circ}$ 9, vol. 6, p. 256), de même que la mutation msd (mutation fauxsens $\mathrm{A} 242 \mathrm{~V}$ ) ont un retentissement clinique sévère avec destruction des oligodendrocytes, absence presque complète de myéline, et mort des souriceaux trois à quatre semaines après la naissance. En revanche, l'allèle rumpshaker [1], comme l'allèle pt (paralytic tremor) chez le lapin, ont des conséquences beaucoup moins graves, avec préservation des oligodendrocytes et survie prolongée.

Chez l'homme, deux maladies cliniquement très différentes: la maladie de Pelizaeus-Merzbacher (PMD), leucodystrophie progressive à début précoce [3], et la paraplégie spastique liée à l'X (SPG2) [4,5], sont dues à des mutations du gène PLP situé en $\mathrm{Xq22.} \mathrm{Mais,} \mathrm{tandis} \mathrm{que} \mathrm{dans} \mathrm{la} \mathrm{PMD,}$ dont le modèle animal est le phénotype Jimpy, les mutations du gène retentissent sur les deux isoformes de la protéine, PLP et DM20, les mutations observées dans la paraplégie spastique n'entraînent qu'une atteinte de la structure de PI.P et épargnent l'isof orme DM20: comme dans l'équivalent murin rumpshaker, les oligodendrocytes sont indemnes. Il importait donc de connaître les rôles respectifs de ces deux isoformes dans des conditions normales et pathologiques. D'autant plus que, dans la maladie de Pelizaeus-Merzba- cher, la variabilité clinique est telle que l'hypothèse de l'existence d'autres gènes situés aussi dans la région Xq22 fut même envisagée. A côté de la forme classique dans laquelle la maladie évolue jusqu'à la deuxième ou la troisième décennie de la vie, il existe en effet une forme congénitale, encore plus sévère, dans laquelle les signes sont présents dès la naissance et où la mort survient avant la dixième année [6]. On est en mesure aujourd'hui d'affirmer que les différentes mutations du seul gène $P I P$ suffisent à expliquer les trois formes, classique, intermédiaire et congénitale de la maladie de Pelizaeus-Merzbacher. Les études, par l'équipe de Lazzarini, à New York, sur la topologie des deux isoformes, PLP et DM20, permettent à présent de comprendre pourquoi les oligodendrocytes, qui sont détruits en grand nombre chez la souris Jimpy ou msd, ne le sont pas chez la souris rumpshaker [7]. Car c'est le degré de l'atteinte des oligodendrocytes qui explique, en partie du moins, la différence de gravité des phénotypes.

Pendant la période de myélinisation du SNC, les analyses morphométriques montrent que les oligodendrocytes ne synthétisent pas moins de $10^{5}$ protéines de myéline par minute. On conçoit que la moindre perturbation dans les voies sécrétoires puisse avoir des conséquences rapidement catastrophiques. Une protéine anormale, ne pouvant être transportée, va s'accumuler rapidement. Même si la protéine est normale, la surexpression du gène $P I P$ peut entraîner une accumulation pathogène. On en a la preuve puisque certains cas de PMD sont dus, non pas à une mutation, mais à une duplication du gène $P I P$ normal [8] et que, chez la souris transgénique porteuse de copies sur- numéraires de Plp doublant la production d'ARNm, on obtient les mêmes lésions du tissu nerveux [9]. Pour pouvoir observer la production, le transport cellulaire des deux isoformes PLP et DM20, et leurs éventuelles interactions, les auteurs ont opté pour les cellules COS-7, transf ectées avec des plasmides codant pour $\mathrm{Plp}^{\text {mst }}$ ou $\mathrm{Plp}^{\text {rsh }}$ ou pour la protéine Dm20 normale, (l'étude des oligodendrocytes in vitro aurait malheureusement présenté trop de difficultés techniques). I.e transport intracellulaire dans les différentes lignées, observé grâce à un microscope confocal, permit les constatations suivantes: la PI.P normale traverse la voie sécrétoire jusqu'à la surface cellulaire, puis entre dans la voie endocytaire pour atteindre les lysosomes périnucléaires. En revanche, les Plp mutées, $\mathrm{Plp}^{\text {msd }}$ ou $\mathrm{Plp}^{\text {sh }}$, ne vont pas au-delà du réticulum endoplasmique. La Dm20 normale ainsi que la Dm20 ${ }^{\text {sh }}$ traversent la voie sécrétoire et prennent la voie endocytaire comme la Plp normale. Mais l'analyse en immunofluorescence des cellules transfectées révéla, en outre, un fait important: la DM20 normale, ainsi que la Dm $20^{\text {rh }}$ sont capables de faciliter le transport de Plp $^{\text {msd }}$ alors que Dm20 msid ne le peut pas. Lorsque la Dm20 est normale, elle réduit l'accumulation de PLP dans le réticulum endoplasmique des oligodendrocytes et limite donc l'atteinte de ces cellules. L'épissage alternatif de l'exon 3 du gène PIP, dont les 105 dernières paires de bases ne codent que pour la protéine protéolipidique PLP, confère à cette dernière une plus grande sensibilité. Certaines mutations vont modifier profondément sa structure alors qu'elles auront peu de conséquences sur l'isoforme DM20. L'analyse effectuée avec les mutations 


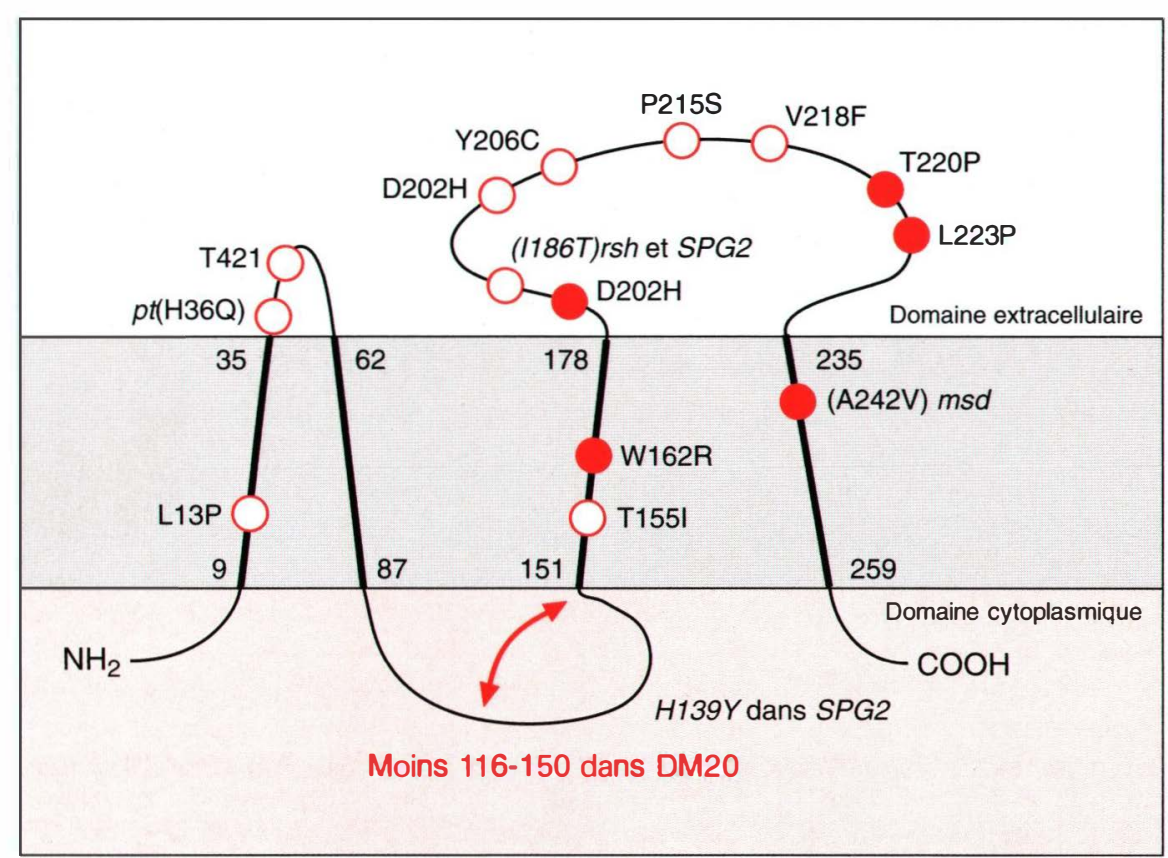

Figure 1. Topologie de PLP et de DM20. L'épissage alternatif de l'ARNm donne l'isoforme DM20 avec 35 acides aminés en moins dans la région cytoplasmique centrale de PLP (région entourée par deux flèches). Les mutations des patients avec une forme congénitale sont représentées par des cercles rouges. Les mutations des patients avec la forme classique sont représentées par des cercles clairs. Les mutations de la paraplégie spastique sont en italique. Les mutations animales sont notées entre parenthèses. La position de l'acide aminé muté est indiquée pour chacune des mutations. pt: paralytic tremor (lapin); rsh: rumshaker (souris); SPG2: spastic paraplegia (homme); msd: myelin sheath disease (souris) (d'après [3] et [7]). A: Ala;C: Cys; D: Asp; F: Phe; H: His; I: Ile; L: Leu; P: Pro; Q: Gln; S: Ser; T: Thr; $V: V a l ; Y: T r p$.

des malades atteints de formes classique et congénitale va dans le même sens. Les protéines DM20 des PMD congénitales s'accumulent dans le réticulum endoplasmique, sans doute par défaut de repliement. On les trouve aussi dans l'appareil de Golgi mais elles sont incapables d'atteindre la surface cellulaire et ne sont pas associées aux marqueurs lysosomaux. Les DM20 des formes classiques s'accumulent dans l'appareil de Golgi et sont présentes à la surface cellulaire et dans la voie endocytaire, de telle sorte qu'on ne peut trouver de différence avec la circulation des protéines DM20 normales.

Ainsi les variations cliniques dépendraient de la capacité d'intervention de la protéine DM20. Cette propriété de faciliter la circulation de la

DM20 car aucune des protéines de membrane (glycoprotéine myéline oligodendrocyte ou MOG, glycoprotéine associée à la myéline ou MAG, entre autres) normalement présentes dans la myéline ne s'est révélée capable de transporter la PLP mutée. Elles furent toutefois retrouvées en abondance à la surface cellulaire, démontrant que la voie sécrétoire n'est pas obstruée en dépit de l'accumulation de protéines anormales dans le réticulum endoplasmique.

Dans la synthèse de D. Pham-Dinh et al. [3], cette interaction entre DM20 et PLP avait déjà été pressentie; le recensement topologique des mutations animales et humaines (figure 1) doit être poursuivi.

S.G.
1. Schneider A, Montague P, Griffiths I, Fanarraga $M$, Kennedy $P$, Brophy $P$, Nave KA. Uncoupling of hypomyelination and glial cell death by a mutation in the proteolipid protein gene. Nature 1992 ; 358: 758-61.

2. Tosic M, Dolivo M, Domanska-Janik K, Matthieu JM. Paralytic tremor $(p t)$ : a new allele of the proteolipid protein gene in rabbits. $J$ Neurochem 1994; 63: 2210-6.

3. Pham-Dinh D, Nussbaum J, Popot J, BoespflugTanguy O, Landrieu P, Dautigny A. Mutations du gène codant pour les protéolipides de la myéline (PL.P et DM20) et démyélinisations liées au chromosome X. médecine/sciences 1992; 8: 664-72. 4. Saugier-Veber P, Munnich A. La maladie de Pelizaeus-Merzbacher et une forme de paraplégie spastique liée à I'X sont toutes deux associées au gène PIP. médecine/sciences $1994 ; 10: 487-8$.

5. Kobayashi H, Hoffman EP, Marks HG. The rumpshaker mutation in spastic paraplegia. Nature Genet 1994; 7: 351-2.

6. Renier WO, Gabreels FJM, Hustinx TWJ, Jaspar HHJ, Geelen JAG, Van Haelst UJG, Lommen EJP, Ter Haar BGA. Connatal Pelizaeus-Merzbacher disease with congenital stridor in two maternal cousins. Acta Neuropath 1981; $54: 11-7$.

7. Gow A, Lazzarini RA. A cellular mechanism governing the severity of Pelizaeus-Merzbacher disease. Nature (jenet 1996; 13: 422-8.

8. Inoue K, Osaka H, Sugiyama N, Kawanishi C, Onishi H, Nezu A, Kimura K, Kimura S, Yamada Y, Kosaka K. A duplicated PI.P gene causing Pelizaeus-Merzbacher disease detected by comparative multiplex PC.R. Am J Hum Genet 1996; 59 : 329.

9. Readhead C, Schneider A, Griffiths I, Nave KA. Premature arrest of myelin formation in transgenic mice with increased proteolipid protein gene dosage. Neuron 1994; 12: 583-95.

\section{Accès à la base de données internationale en Immunogénétique : IMGT}

La base de données internationale ImMunoGeneTics, IMGT, initiée et coordonnée par Marie-Paule Lefranc Montpellier, France, est accessible sur le serveur WWW du CNUSC (http:// imgt.cnusc.fr). IMGT comprend deux bases de données : LIGM-DB (Immunoglobulines et Récepteurs T) et MHC/HLA-DB. IMGT/LIGM-DB contient à ce jour plus de 19000 séquences (13 798 séquences d'Immunoglobulines et 5850 séquences de récepteurs T) de 78 espèces différentes. Les fichiers à plat sont accessibles sur le serveur ftp anonyme d'EMBL-EBI (ftp.ebi.ac.uk/pub/ databases/imgt).

Contact:

Prof. Marie-Paule Lefranc, Coordinateur de IMGT Tel. : +33(0)467613634 - Fax : +33(0) $467040231 / 45$ E-mail : lefranc@ligm.crbm.cnrs-mop.fr 